\title{
A NEW EXPLANATION FOR THE ILLUSORY MOVEMENTS SEEN BY HELMHOLTZ ON THE ZÖLLNER DIAGRAM.
}

\author{
DR. A. H. PIERCE.
}

Amherst College.

Every one who has given any considerable attention to the Zöllner illusion is familiar with the strange gliding movements of the vertical columns which, under special circumstances, are

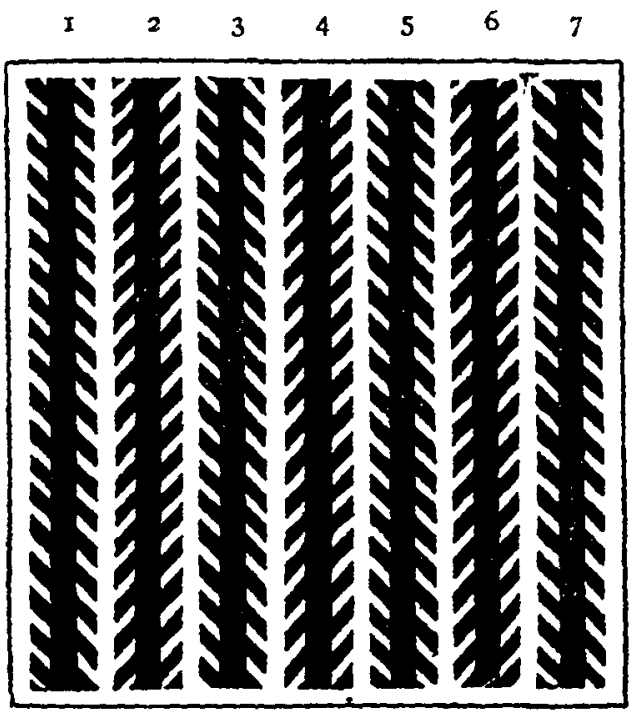

FIG. I.

to be seen on the heavy-line pattern here figured. (Fig. I.) Helmholtz was the first to describe these movements. ${ }^{1}$ According to him, they are to be seen where one fixates the point of a needle moved horizontally across the diagram. If one be sufficiently practiced in following steadily a moving point a most striking and unusual phenomenon is presented. The vertical

1 Physiologische Optik (2), 712. 356 
columns are seen to shift their position with a graceful gliding movement in the direction of their length. Those verticals that bear upward-running transversals glide in one direction, while those with downward-running transversals pass as rapidly in the opposite direction. If the movement of the fixated point be from left to right, the former columns (2, 4 and 6 of Fig. I) dart upwards, the latter downwards, just the reverse being the case when the moving point goes from right to left. Moving the fixated point alternately back and forth, and taking care to maintain an appropriately moderate rate of speed, there results a state of 'strange unrest' over the whole diagram, totally unlike anything else in the whole realm of geometrical optical illusions. One is reminded most forcibly, perhaps, of what might happen if the illusory creeping motion of the threads of rapidly rotating endless screws were presented to the eye in seven parallel lines and with alternate differences of direction. Not every one gets this illusion directly, but a brief practice in moving fixation, as well as some attention to the most favorable rate of movement and distance of diagram from the eye, will reveal it in all its vividness.

Now as to the existence and general nature of this illusion there can be no question. The apparent slippings and slidings of the columns are incontestable. It is only as to their explanation that there may be disagreement and discussion. It is accordingly the purpose of this paper to offer an entirely new explanation for these phenomena, an explanation which is based upon a consideration of the peculiar manner of stimulation experienced by the retina as the fixated eye passes over the diagram. That is, the explanation here given will be couched wholly in retinal terms, and not in terms of any elementary geometrical illusions which arise under the given conditions and which themselves cause the phenomena in question.

But let us see first what explanations are in the field. I have been able to find three: (a) Helmholtz's own is as follows: 1 The path of the moving eye is along a line which makes an acute angle with the oblique transversals which it crosses; or, more generally stated, every sensitive point of the

'Loc. cit., 7ז2. 
retina is moving in a direction oblique to the actual obliques. There results a multitude of ideal acute angles, or " directiondifferences," and since acute angles are always enlarged for perception, or what amounts to the same, since directiondifferences are always magnified by contrast, the numerous transversals must appear to swing in such a way as to express this illusory angular enlargement. The upward-running transversals will consequently swing upwards, the downward-running downwards, and since any given system of transversals will move in concert, this illusory movement must be comnunicated to the vertical strips themselves. This is the substance of Helmholtz's explanation. It rests, plainly, upon a supposed angular illusion, the production of which draws the further illusion in its wake. Some of the minuter considerations, which in Helmholtz's view go to support this explanation, must be carefully examined later.

(b) Thiéry ${ }^{1}$ claims to find the cause of these movements in the equivocal character of the prism surfaces upon which, to many observers, the vertical strips of the Zöllner pattern seem to be projected. To those gifted with the power to see perspective effects in linear drawings, these prism surfaces appear to tilt alternately backwards and forwards, and thus, on the perspective theory of optical illusions, the ordinary Zöllner illusion is produced. In the usual forms of the figure the intersections of these prism surfaces must of course be imagined lines lying in the white verticals between the black columns. Now these edges of intersection being imagined, as the eye moves horizontally over the figure, each successively fixated edge, Thiery says, steps into the foreground-as fixated points or lines of equivocal figures always do-and thus each imagined intersection becomes in turn the. front edge of a prism. By an immediate consequence the adjacent intersection on either side must retreat into the background, only to step forward once more when the moving fixation-point has reached the next intersection. It is this ceaseless movement backwards and forwards, consequent upon the movement of the eyes and due to the equivocal nature of the figure, that to Thiery's mind is responsible for the 'strange unrest.'

'Phil. Studien, 1895, XI., 320-321. 
Filehne ${ }^{1}$ seems to entertain an identical opinion of the matter, though the expression of his opinion is nowhere explicit.

(c) Judd ${ }^{2}$ adopts still another point of view by attempting to apply the observation of Müller-Lyer that if an acute and an obtuse angle have equal legs, those of the obtuse angle seem longer. Now, he says, the transversals of the Zöllner pattern make both acute and obtuse angles with the intersected verticals, and the horizontal movement of the eye, allowing, as it does, the successive fixation of the various points of the figure, permits the successive false estimations of the sides of these angles to come into prominence. Of course the mal-estimations that are effective for this illusion are those connected with the legs formed by the verticals themselves. And the particular slope of the transversals of a given column will determine the direction, up or down, in which this column will appear to move.

It will be seen that Helmholtz alone attributes any influence to the imaginary line drawn over the figure by the moving eye. The other two writers look upon the eye's movement merely as necessary to bring out characteristics latent in the figure, the conditions being thereby supplied for the arising of the further illusion of the gliding columns.

It is really remarkable that this curious illusion of motion has received so little serious attention. Either because this is less easy to see than the usual illusion that has engaged the almost exclusive attention of observers, or because the weight of Helmholtz's authority has tended to confine explanatory attempts within a particular realm of spatial phenomena, the true cause of this illusion, simple and near at hand as it is, has been persistently overlooked.

\section{An Explanation in Terms of Peculiarities of Retinal Stimulation.}

Suspicion against the current explanations may be readily aroused by noticing that the illusory movements are only faintly perceived upon the light-line models of the Zöllner figure. Attention is always directed to the original heavy-line diagram,

${ }^{1}$ Zeitsch. f. Psychol., etc., I898, XVII., 47-48.

'PSPCBOLOGICAL, REVIEW, I899, VI., 260. 
when this matter is under discussion. This would seem at once to indicate that purely retinal influences are powerfully operative in determining at least the vividness of the illusion.

My own suspicions were first awakened by accidentally noticing what happened when, with the diagram in full view, the convergence of the eyes was unthinkingly relaxed. As the eyes diverged the illusory movements began. They occurred again while the eyes were returning to converge upon the diagram. And if, when the optical axes were nearly parallel, there were slight movements to a state of greater or less divergence, the columns exhibited on their part their appropriate shiftings. In themselves, of course, these observations contributed nothing decisive to the problem in hand, but they served to arouse a line of investigation which has not been altogether without positive results.

It may be said here that for ease of observation a copy of the Zöllner pattern, like that of Fig. I, should be pasted upon a small piece of cardboard of convenient size for handling. With this device one may demonstrate the Helmholtz phenomenon much more readily than by the usual method. Holding the column in any desired position-vertical, horizontal or at any angle-it is only necessary to shake the diagram slightly back and forth in its own plane and in a direction perpendicular to that of the columns to produce very vivid effects. The 'shaking' of the diagram is essentially the same as moving the eyes over it, for the eyes cannot readily follow its motions and consequently the image of the diagram moves over the retina, the result being the same for perception as if the retina moved over the diagram. The relations between objective and illusory movements in respect to their directions are the same with this as with the usual method of producing the illusion. The slippings and slidings of the columns to be seen under these circumstances strongly suggest that we are in the presence of phenomena analogous to those of the 'Fluttering Hearts.' There is a similar jelly-like movement here, but instead of a dim illumination the full daylight is requisite for the best effects. That the observation of the illusion is possible by the method just described would seem at least to show that Thiéry's explanation in 
terms of the equivocal perception of prism-faces cannot find any reasonable application under the conditions here in force. No trace of such equivocal swingings of the prism surfaces is to be discovered.

Further, it becomes hard to reconcile the theory of Helmholtz with the observation that the columns move in such a way that the obliques are everywhere parallel to their original direction. That is, if the apparent movement of the columns is due to a principle of direction-contrast working upon the oblique transversals, or, in other words, if this movement is due to the illusory enlargement of acute angles successively formed by ideal lines passed over by the moving eye, there should be some vestige of a rotary motion observable on the obliques. For angular increase must take place about a vertex as center of rotation. And if such angular increase is the underlying cause of the phenomenon under consideration, it surely must be possible to perceive some twist in the transversals, some slight departure from their original direction. But as a matter of fact no such thing is to be seen. The columns move smoothly and evenly upwards and downwards, the obliques never changing in the slightest the course of their original slope. They rotate neither about the point of intersection with the verticals, nor about either of their ends. They move rather as if impelled by some push given to the verticals, to which they seem rigidly attached. If correct, this observation most certainly discredits Helmholtz's explanation.

And, finally, Judd's explanation in terms of mal-estimations of sides of angles is just as little able to maintain its claims. For the verticals are not at all necessary for the illusory movements. By cutting suitable strips from striped cloth or from properly ruled paper, the Zöllner pattern may be reproduced without its verticals. Under these circumstances the Helmholtz illusion persists unchanged in all particulars. But Judd's explanation is no longer applicable, for the sufficient reason that there are now no acute or obtuse angles whose sides are to be successively mal-estimated as the eye moves over the diagram. The main lines which supplied the indispensable condition for this explanation are no longer present, and it seems hardly 
reasonable to suppose that imagined verticals may take the place of the actual lines that have been removed.'

It being now evident that no one of the current explanations can square with the simplest facts of the case, let us attempt the statement of the purely retinal theory promised above. Consider, for convenience, a single column of upward-running

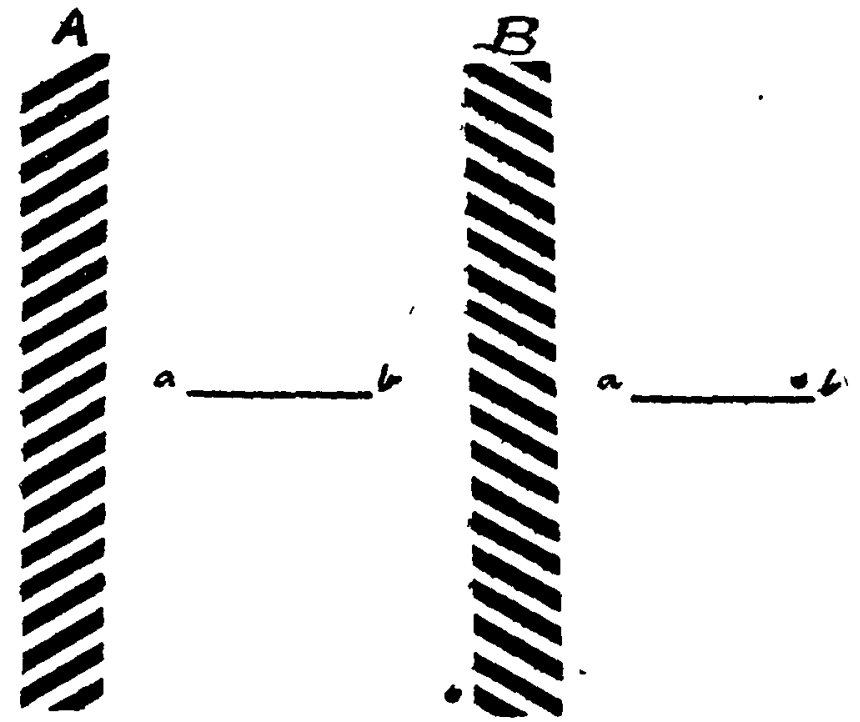

FIG. 2.

obliques without the central vertical ( $A$, Fig. 2), and let the eyes be supposed to follow a point moving horizontally from left to right. That the illusion persists with only a single column may be easily ascertained by carrying out the usual movements of the eyes after all but one of the columns of the reg-

1The observation of Witarek (Zeitsch. f. Psychol., etc., I8g8, XIX., I53) to the effect that he observed no movement of the columns when the obliques alone were presented to one eye, though such movements were continually being observed on the complete diagram upon any slight movement of the eyes, was probably due among other things to the lightness of the lines he was observing. He was making a study of the usual Zollner illusion under the conditions of stereoscopic combination-the verticals being presented to one eye and the obliques to the other-and under these circumstances the attention was presumably too fully occupied with other matters to notice any faint illusion. If, however, the eye had been moved intentionally over the part bearing the obliques only, the illusory movements could not have escaped his notice. 
ular pattern have been covered. As to the position of the fixated point, experience shows that the clearest perceptions emerge when it moves along a line situated at the side of the column, along the line $a b$, that is, in Fig. 2. For my own eyes the most satisfactory results are obtained when this line is at the right. Others may find that the left side is preferable. In any case indirect vision seems best adapted to the perception of the illusion.

What happens under these circumstances, as the eyes follow the fixation-point along $a b$, may best be seen by considering the experience of a single vertical line of retinal elements. Let this line be thought of as resting on the extreme left edge of the column before us. It will then receive along its length alternate excitations of black and white. Now let the eyes move. As the supposed line of retinal elements passes from the left to the right edge of the column, the alternate excitations of black and white will fall higher and higher upon the line. The practical result for perception is therefore exactly as if numerous stimuli had moved up over a resting retina. This line of elements has experienced the full conditions for the perception of motion. But what happens to the single line happens in like manner to all vertical lines of elements that pass over the column of obliques, the upward-creeping excitations on each succeeding line being in each case lower down than those on the line ahead, as is of course determined by the slope of the stimulus-giving obliques. Manifestly the visual effect of this is entirely similar to the moving of the column bodily upwards over the resting eye.

If now the eyes move from right to left, the vertically arranged spots of stimuli, as we have pictured them, will travel downwards, and the visual appearance will be that of a descending column of obliques.

The directions of these vertical movements, it will be noticed, are in exact accord with the observations of Helmholtz. Columns bearing upward-running transversals run upwards when the eyes move to the right, downwards when the eyes move to the left.

Exactly the same style of considerations is to be applied to 
the columns of form $B$ (Fig. 2). With a movement of the eyes to the right successive stimulations will fall upon lower and lower points of the retina and the column as a whole will appear to move downwards, just the reverse being the case when the eyes move to the left. This again is wholly in accord with Helmholtz's observations.

The conclusion of the whole matter then is simply that the illusion under discussion is caused by the peculiar manner in which stimulations travel upon the retina. The horizontal movement of the eyes across lines lying oblique to their direction is equivalent in retinal terms to an ascending movement of the entire set of obliques over a resting retina. For perception the two processes have identical effects. Hence the illusion.

The general correctness of the view just expounded may be vividly brought out by the following procedure. Taking any piece of cloth or paper with closely lying stripes and placing upon this a piece of cardboard in such a way that the stripes run upwards across the vertical edge, move the cardboard in a direction perpendicular to its edge, the eyes meanwhile following some point on the latter. The effect will be that of an apparent ascending of the portions of the cloth successively uncovered by the moving cardboard. What occurs here is essentially the same as in the typical cases. Stimulations are mounting higher and higher along the vertical lines of the retina, and hence all the usual conditions for the perception of upward motion are fulfilled.

If the above explanation is correct, several important corollaries follow immmediately from it:

I. The rate of the illusory movement must depend upon the rate of the horizontal movement of the eyes. Helmholtz does not fail to notice this fact, ${ }^{1}$ though he simply mentions it without discussion. It may be readily verified on the regular 'pattern' or upon isolated columns of obliques. Now on the theory of moving retinal stimulations this coincidence of rates must occur. The more rapid the horizontal movement of the eyes, the more rapidly will any bit of stimulus traverse its path rom end to end of any ob lique, and consequently the more illusory speed will the vertical column seem to possess.

'Loc. cit., p. 713. 
2. The excursion of the illusory movement must depend directly upon the slope of the oblique. This again is evidently a direct consequence of our explanation, for the vertical distance from tip to tip of each oblique increases with the slope of the latter, and accordingly the amount of vertical movement of the various stimulations along any retinal line, such as we have supposed above, must vary directly with the

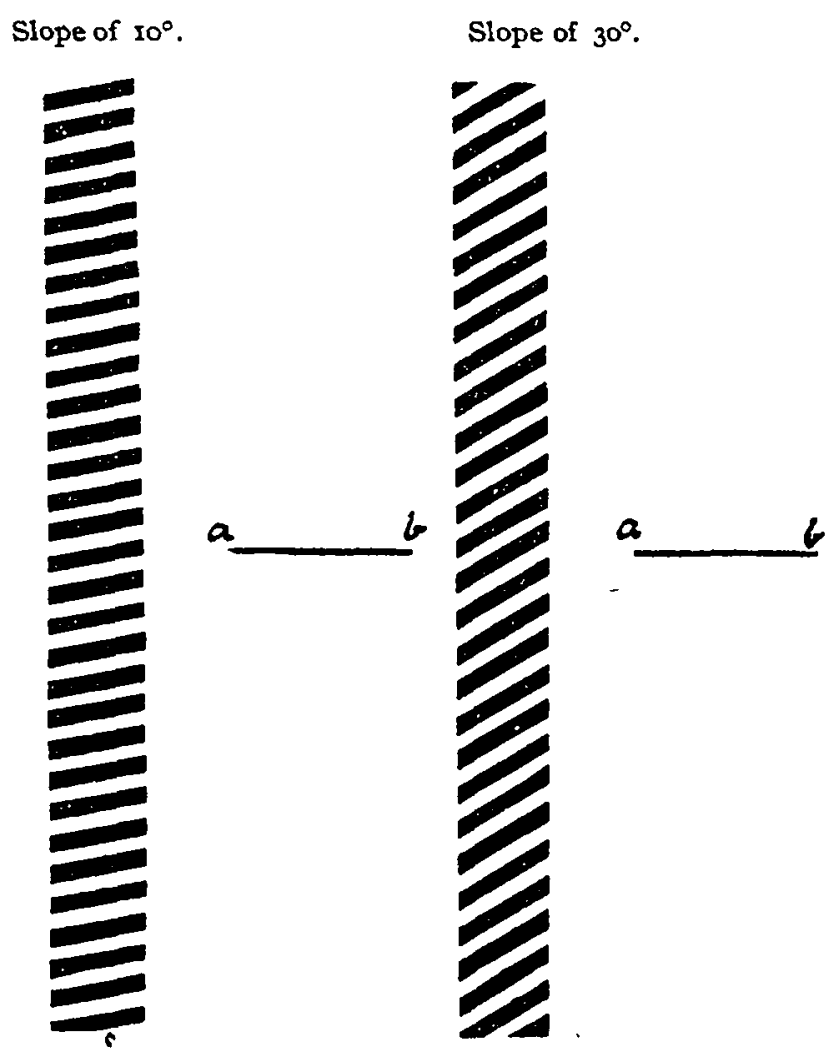

Fig. 3 .

tilt of the oblique. To subject this deduction to the experimental test, cards were prepared bearing columns whose obliques sloped at angles of $10^{\circ}, 20^{\circ}, 30^{\circ}$ and $40^{\circ}$ respectively. At the side of each card, in the position indicated in the accompanying figure (ab, Fig. 3$)$ a slit was cut which allowed the introduction from above of a common pin. The head of 
the pin, moving upon the surface of the card, served as moving fixation-point for the eye. The length of each slit was $18 \mathrm{~mm}$. Naturally the results obtained do not admit of quantitative statement, but the anticipated increase of the illusion with the increase of the slope was everywhere realized. The most striking way to bring this into evidence is to 'shake' the cards, in the manner described on page 360 , the columns being held horizontally. There is no question that the columns whose obliques have a slope of $40^{\circ}$ appear to traverse a much greater horizontal distance than those with $10^{\circ}$ obliques.

Now it is difficult to see how Helmholtz's explanation can adequately account for this fact. Indeed it would seem that Helmholtz must expect exactly the reverse of what we have found. For in accordance with his principle that clearly recognizable differences are estimated as greater than those less clearly recognized, ${ }^{1}$ it would seem that the angular enlargement due to direction-contrast would be greater for a slope of $10^{\circ}$ than for one of $40^{\circ}$. For in the former case the difference of direction between the obliques and the imaginary paths traced by the eye must be clearer than in the latter case. Or, if not clearer, at least of a similar grade of clearness, which should cause the illusion to be equally great in the two cases. But it is unnecessary to attempt to strain Helmholtz's explanation into any form which shall pretend to cope with these facts in regard to the amount of the illusory displacement of the columns. These facts fall so readily into line with the retinal explanation proposed that they form one of its strongest confirmations, just as their absence would completely overthrow the theory here defended.

(c) Again, on the view here presented, the illusory movements should progress smoothly, without the slightest change of inclination on the part of the obliques. We have already emphasized the fact that this is so. And, in addition to the above, any slight movement of the eyes along the line of fixation should give a correspondingly slight illusion. And movements of the eyes by stages, that is with momentary stops between short movements, should be accompanied by an exactly parallel be-

${ }^{2}$ Loe. cit., 705 and 714. 
havior of the columns. Both of these deductions are unmistakably verified. Especially may one see what is meant here by substituting for the column of Fig. 3 a rather large patch of oblique lines. The movement of the eyes by tiny stages makes the patch appear to move stealthily up and down, creeping slightly along, then resting, then creeping further, etc. The whole impression during such an observation as this is that of some set of visual stimulations making their way gradually up and down the retina. Of the rotation of the obliques, which if it existed should assuredly become evident with these relatively long lines, not a trace is to be observed. There is the same smooth upward march that is to be seen in the case of the usual columns.

Another way of demonstrating this same thing is by diverging and converging the eyes upon the diagram as described above. This experiment may be varied by taking a single column from the 'pattern.' As the axes of the eyes diverge towards parallelism two columns are of course seen moving in opposite directions. But what interests us here is that every chance alteration in the degree of divergence produces a corresponding movement of the columns; slow, if the change in the eyes is slow, and of short excursion, if the change of divergence is slight. In short, when the eyes move there are immediate response and complete correspondence on the part of the moving column.

Now these facts can hardly be made harmonious with the thought of direction-contrasts that develop as the eyes move. Surely some length of movement must be requisite for the development of the consciousness of such direction-contrasts, and one should expect on this hypothesis, not the smooth movement that we have observed, but rather the progression of the columns by jerks, as the ideal horizontal line became long enough to evoke a feeling of difference in direction. Such jerky movements might not reveal themselves under the usually observed conditions of continuous eye-movement, but it would seem plausible at least to expect them under the special conditions here in force, were Helmholtz's explanation correct. The entire absence of such unsteady motion in the illusion, and the total impossibility 
of discovering the faintest trace of rotation on the part of the obliques, combine to form a strong bit of evidence against the explanation here combated.

(d) That columns of upward-running obliques should run upwards, and that columns of downward-running obliques should run downwards, is an inevitable deduction from our premises.

Now Helmholtz records this fact with perfect clearness, but for some strange reason he does not attempt to show why either sort of column should have its peculiar direction of movement. And indeed on his theory this would be an awkward task. For instance, consider a single upward-running oblique. If the imaginary path, which is to establish the direction-contrast, be supposed to cut the oblique at its point of intersection with the vertical, the oblique must execute opposite movements with its two ends. With the eye passing to the right, the left end of the oblique must move down, the right end up. Which of these two movements is to be decisive for the final movement of the column? The one last seen? But why should not the movement first seen be just as effective, and consequently why should not the opposite sides of each column move in opposite directions? But there is a deeper difficulty. We have no basis for supposing that the imaginary path intersects the oblique at any particular point. All the sensitive points on one side of the retina are sweeping over the oblique and are cutting it at all conceivable places. Therefore, if for convenience we choose to mention a special point of intersection, we have no right to select one that is favorable to the result desired in preference to one that is absolutely unfavorable. For example, in our supposed case, we are in no way warranted in placing the point of intersection at the left end of the oblique rather than at the unfavorable right end. Under the former circumstances the angle would enlarge upwards, in the latter case downwards. In the one case we should have found an apparent explanation; in the other we should have met with a blank contradiction. The truth is that Helmholtz's theory is simply inapplicable. And may it not be that his silence in reference to the cause of particular directions of the illusory motions is not without important significance? 
Though the evidence now adduced in favor of the purely retinal hypothesis may be regarded as sufficiently conclusive, one or two further points may give added weight.

The illusion does not always appear instantaneously when the eyes begin to move. A certain degree of retinal fatigue seems requisite for the full intensity of the phenomenon. This may be most effectively obtained by a moment's steady fixation of the point before beginning the movement of the eyes. This secures a slight after-image and provides a retinal point of reference for the ensuing movements of the various stimulations, and the illusion attains its most lively form.

At the same time a good illumination is a prime necessity. In a dim light the movement of the fixated point must be much slower than in a strong light, and the illusory movements are thereby greatly reduced in vivacity. Or if the movement of the eyes be the same for the two conditions of illumination, the illusion will almost completely vanish with a degree of illumination that is still sufficient for the clear perception and distinct discrimination of the lines when the eyes are at rest. Thus we find that the most advantageous conditions for the illusion itself are precisely those which are conducive to the most marked sensational effects.

A further point of interest is the heightening effect of contrasting illusory movements simultaneously present. In the Zöllner pattern these contrasts are already provided for, since alternate lines are moving in opposite directions. An isolated column, however, whether taken from the 'pattern' or constructed without the central vertical, gives an illusion of greatly diminished liveliness. Consequently in the experiments above described it is much more satisfactory to have a column of oppositely sloping obliques-most conveniently taken from the ' pattern'-ranged alongside of the particular column under investigation.

A peculiarity about these contrasting effects is that in the majority of cases only one column seems to move. This one column absorbs, as it were, and appropriates to itself whatever motion the other may have in the opposite direction. In my own case it is the nearer column which appears to stand still. This seems 
to discharge its functions by acting as a reference column. As such, however, it undoubtedly contributes strikingly both to the clearness and to the apparent excursion of the illusory motion of its companion. This effect of contrast is prettily demonstrated by taking any pair of adjacent columns from the 'pattern' and then comparing the vividness of the illusion on that column which exhibits movement with the relatively feeble illusion resulting when the 'quiet' member of the pair is replaced by a column of short horizontal lines. The latter column might indeed be supposed to furnish a basis of reference, but its lack of illusory movement makes it practically without effect upon its companion.

It is, presumably, this principle of contrast which must be appealed to in explanation of certain minor phenomena to be observed when the complete diagram is 'shaken' before the eyes. Holding this in the horizontal position and shaking with a quick, short motion of the hand, all columns fall into their appropriate movements. But if the shaking movement be somewhat longer and less rapid, only columns 2,4 and $6^{1}$ will present the illusion. Now and then, possibly, the other columns will momentarily take on motion, but in general they seem to play the reinforcing rôle. That columns 2,4 and 6 should display the illusion seems to depend upon the fact that they are most advantageously situated within the set of columns for receiving the full influence of contrast. That it does not depend upon the kind of the column, that is, upon the sort of slope that the obliques of 2,4 and 6 happen to have, is shown by the fact that if a diagram be constructed by replacing each column by its opposite, the illusion will still be confined-at least most vividly-to the columns occupying the same positions as 2,4 and 6.

It remains now to examine those widely accepted elementary phenomena upon which Helmholtz principally based his explanation. I refer to those oft-quoted cases, which the accompanying figure, Fig. 4, will call to mind immediately, where a direction-contrast, developing under the very eyes, graphically

'See Fig. I. 
performs its work of producing the overestimation of an acute angle.

Helmholtz's claim was, as every one knows, that, if the end of one leg of a pair of compasses be followed by the eye as it moves over the path $C D$, across the line $A B$, the two halves of the line will appear respectively to assume the positions indicated by the lines $a a^{\prime}$ and $b b^{\prime}$. That is, the path of the com-

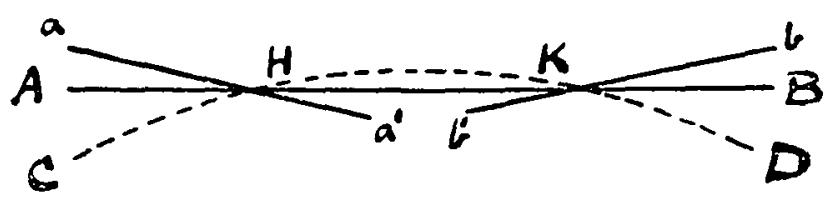

FIG. 4 .

pass-point has made acute angles with the intersected line, these have been perceptually enlarged, and the left and right halves of $A B$ have been seemingly forced out of position in the process of accommodating themselves to this unavoidable and insurmountable illusion of space perception. At first sight the phenomenon appears to be the isolated element of the illusory movements that we have been discussing above. The path of the compass-point as it cuts the line is the representative of the many paths swept out by the retinal elements as they intersect the obliques of the Zöllner pattern. And as either half of $A B$ in Fig. 4 swings about the point of intersection, so the intersected obliques of the 'pattern' swing about and give the appearance of vertical motion to the columns. We have already seen plenty of reasons for rejecting this interpretation as applied to the larger phenomenon. And if now we are able to find some other interpretation for this supposed elementary illusion, all possible grounds for clinging to the explanation of Helmholtz will have been taken away. That an illusion of direction is to be seen when the conditions of Fig. 4 are fulfilled is unmistakable. But a really complete description of what happens seems never to have been given. For the more convenient study of the matter a heavy line of $4 \mathrm{~mm}$. thickness and $50 \mathrm{~mm}$. length was drawn upon paper and the latter fastened to the wall directly in front of the observer. A slender rod $475 \mathrm{~mm}$. long and tipped with a steel knitting needle swung from a center be- 
low in such a way that the tip of the needle traced an arc 22 $\mathrm{mm}$. above the line at the highest point. This arrangement reproduces all the essentials of the original, while allowing greater facility in the way of control and observation.

What happens to the line $A B$ as the point is carefully followed in its course is certainly not adequately represented by the usual oblique straight lines of Fig. 4. There is rather an apparent sagging of the line at the center, somewhat as represented in Fig. 5. And furthermore this whole appearance of

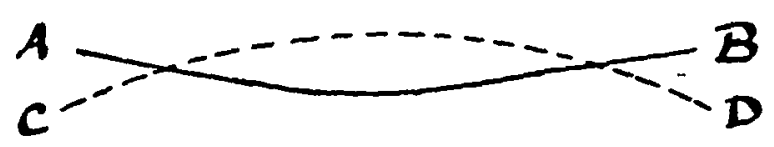

Frg. 5.

distortion may be produced by confining the moving point to the portion of the arc that lies between $H$ and $K$ (Fig. 4). That is, the actual intersection of the line by the path of the moving point is not at all essential to the illusion. This in itself is sufficient to cast an interesting suspicion on the hypothesis of direction-contrasts. For here we are obviously dealing with the path of the fixation-point, and not with ideal lines traced by any point whatever of the retina. A careful examination of the behavior of the line during the movement of the eye from $H$ to $K$ will, I think, reveal the following: If the moving point stop short of the crest of the curve, the line $A B$ seems simply to move downwards, keeping always parallel to its original position. This is a necessary consequence of the eye's movement obliquely upwards, for the perceptual effect, or at least the retinal effect, is precisely what it would be were the line to move downwards and to the left. The sidewise component of the movement is not noticed unless the line has some conspicuous marks upon it. Again, if the moving point pass from a position just beyond the crest of the arc down across the line, the latter will seem simply to move upwards. In neither of these cases is any departure of the line from its original direction perceivable. But if now the moving point be followed throughout its entire course, or even if it be confined to the position $H K$, the sagging of the line appears. But this appearance is manifestly only a 
necessary perceptual interpretation of three contradictory perceptions that come about successively as the point moves. First, while the eye is approaching the summit of the arc the line moves down, then as the summit is being passed it ceases altogether to move, and finally as the eye sweeps downwards the line moves upwards. That is, during the passage of the eye over the length of the curve, the line has presented three successive phases-upward movement, no movement, downward movement. Now bearing in mind that the attention is most sharply given to that portion of the line that is at any moment just below the fixation-point, and considering further that the final perception of the whole line must be made up of these three partial perceptions which follow each other so rapidly as to fuse into a single resultant, the illusory sagging line seems the only possible spatial form that could be perceived. The illusory sagging appears just because three incongruous perceptions are forced into one by the fact that the line $A B$ must be perceived as a continuum, and the three modes of behavior of its three different parts must be apperceived as occurring upon an unbroken, continuous line.

There is no need of appealing to the influence of directioncontrasts here. We are merely in the presence of one of the so-called 'illusions of interpretation.' And as thus viewed this phenomenon has not the slightest connection with those other illusory movements which form the subject of this paper. Different causes are operative in the two cases, though both are reducible to relatively simple retinal happenings and need no underlying spatial peculiarities for their full and satisfactory explanation.

Helmholtz, it will be remembered, claimed to find evidence for the working of a direction-contrast when the path of a moving point is along a straight line-whether actual or ideal is indifferent-cutting a horizontal line at a small angle. The use of the compass-point arc in place of the straight-line path was, according to Helmholtz, simply to secure more vivid results. But I cannot refrain from thinking that Helmholtz's zeal to obtain a preconceived result led him here to fall into error. And I cannot find that others have verified the alleged observation that 
the main line seems to incline away from the path of the moving fixation-point as the latter cuts across it. I have carefully examined the matter, using the same heavy line as above and letting the imaginary oblique be traced by the tip of a stiff wire fastened below to a tiny car, sliding upon an inclined plane. The moving tip was of course close to the plane of the paper bearing the line, and the angle of intersection was $5^{\circ}$. Now with an excursion of the moving point of about $\mathrm{ro} \mathrm{cm}$. on each side of the line, nothing whatever beyond the usual and inevitable up-and-down movements of the line was to be seen. There was not the faintest vestige of tilting of the line. Nevertheless such tiltings were occasionally seen, yet so inconspicuous as to be easily overlooked, when the imaginary line was made as long as the horizontal itself. But this, once more, is only an illusion of perceptual interpretation. As said above in discussing the companion illusion, it is the portion of the line just above (or just below as the case may be) the moving fixationpoint which seems most of all to be in movement. Accordingly when the eye, passing say from left to right, arrives at the end of its path, it is the extreme right end of the horizontal that is at that instant in most vivid apparent movement downwards. The rest of the line is still visible, of course, but it is relatively motionless. What other possible result then for perception than that the horizontal seems to be assuming a slight inclination downwards. The correctness of this view of the case is made overwhelmingly probable, it seems to me, by the fact that the illusory inclination of the line does not appear at about the time the line is being intersected, as we should expect on the theory of direction-contrast, but comes rather at the end of the whole path. Any one who will carefully repeat the experiment will be convinced, I feel sure, that the illusion here is due to the manner of interpreting motions on the retina and not to the over-estimation of angular magnitudes.

It may seem that these elementary phenomena have been dwelt upon at needless length. But I cannot help thinking that a real error has been firmly attached to them ever since Helmboltz first made his communications in respect to them. And since they have been so often referred to and so often made the 
explanatory basis for other illusions which have no actual relationship with them, no amount of pains is too great which shall contribute to a more accurate understanding of their provoking cause.

\section{Summary and Conclusion.}

We have now the various facts and arguments before us. In conclusion these may be gathered together for convenient inspection. To begin with, we saw that no one of the current explanations for the movements to be seen on the Zöllner pattern could stand aright before certain easily verified facts connected with the illusion. That of Thiéry, in terms of equivocally perceivable prism-edges, could not meet the case where the illusion was produced by simply 'shaking' the diagram before the eyes. Grave discredit was seen to be cast upon Helmholtz's explanation by the observation that no change of inclination was to be seen on the part of the obliques during the presence of the illusion, though such change of inclination was supposed by Helmholtz to be the underlying cause of the apparent movements. And finally Judd's explanation was rendered inapplicable by the removal of the central verticals, there being thus no sides of angles to be falsely estimated.

The proposed explanation in terms of peculiarities of the movements of stimulations upon the retina was then supported by several considerations: First, that the rates of the actual and illusory movements correspond; second, that the excursion of the illusory movement depends directly upon the degree of the slope of the obliques; third, that the illusory movements, presenting, as they do, a behavior corresponding perfectly with that of the eye in respect to rest, movement and kind of movement, give the impression always of the passing of visual stimulations along the retina: and, lastly, that the particular directions in which the differently constructed columns move are now for the first time adequately accounted for.

And then finally those alleged basal phenomena in connection with single lines were seen to be entirely capable of a new interpretation, which removes them completely from any kinship with the more complex illusion which Helmholtz supposed them to explain. 
Not only then have the curious movements of unrest that the Zollner pattern may be made to show found an explanation that satisfactorily accounts for all the peculiarities connected with them, but in addition the fundamental basis for the original and most widely accepted explanation has been swept away. This new explanation, by being substituted for the old one, will probably have no far-reaching effect upon the interpretation of other geometrical optical illusions. Nevertheless it is decidedly worth while to get each individual illusion set in its true light. 